\title{
MANAGEMENT OF PORTAL HYPERTENSION IN PAEDIATRIC AGE GROUP
}

\author{
Keshava Murthy Muniswamy1, Hariprasad Taluru Ramachandra Naidu², Raghunath Bangalore Vasudev
}

${ }^{1}$ Associate Professor, Department of Paediatrics Surgery, Rajarajeswari Medical College Hospital.

2Professor, Department of General Surgery, Rajarajeswari Medical College Hospital.

${ }^{3}$ Associate Professor, Department of Paediatrics Surgery, Rajarajeswari Medical College Hospital.

\begin{abstract}
Management of portal hypertension in children has evolved over the past several decades. Portal hypertension can result from intrahepatic or extrahepatic causes. Management should be tailored to the child based on the etiology of the portal hypertension and on the functionality of the liver. It frequently presents with bleeding from esophageal varices. Precise diagnosis, a sound understanding of the therapeutic options and a multidisciplinary approach are essential for successful management.
\end{abstract}

\section{KEYWORDS}

Portal hypertension.

HOW TO CITE THIS ARTICLE: Muniswamy KM, Naidu HTR, Vasudev RB. Management of portal hypertension in paediatric age group. J. Evolution Med. Dent. Sci. 2016;5(9):386-388, DOI: 10.14260/jemds/2016/88

\section{INTRODUCTION}

Formation of portal vein is by the confluence of the splenic vein with the superior mesenteric vein and its formation mostly occurs behind the pancreas in the retro peritoneum. It transports the blood mainly from the gastro-intestinal tract and the spleen to the liver. Seventy percent of the total blood supply to the liver is contributed by the portal vein, while the hepatic artery contributes to the remaining thirty percent. The intrahepatic branches of the portal vein terminate in small vessels that supply the hepatic sinusoids. Embryologically, the systemic veins of our body develop from the intra-embryonic anterior and posterior cardinal veins, while the portal system develops from the extra-embryonic vitelline and umbilical veins which drain from the yolk sac and the placenta.

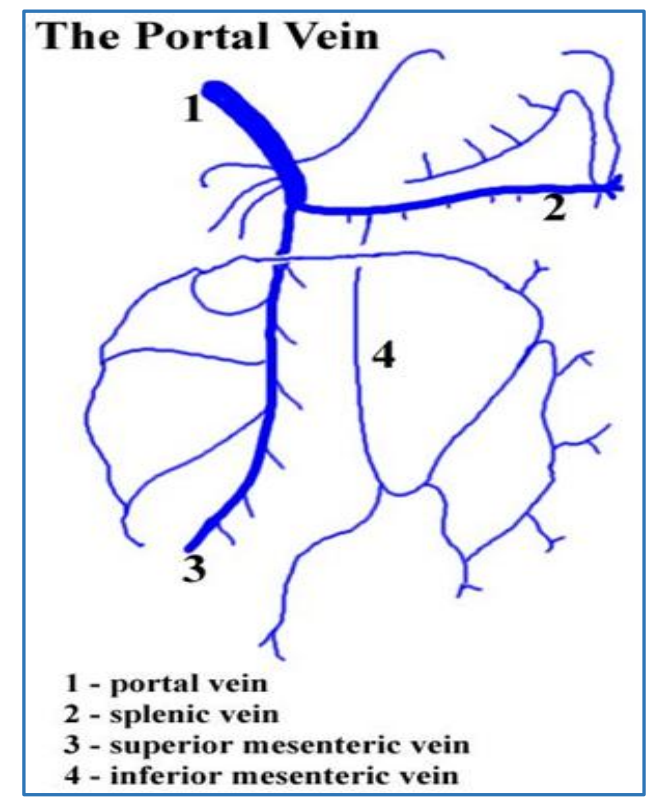

Financial or Other, Competing Interest: None.

Submission 17-12-2015, Peer Review 12-01-2016,

Acceptance 20-01-2016, Published 30-01-2016.

Corresponding Author:

Dr. Keshava Murthy Muniswamy,

$14 / 1,2^{\text {nd }}$ Cross, AT Street Yediyur,

Bangalore-560082.

E-mail: drkeshav_2000@yahoo.com

DOI: $10.14260 /$ jemds/2016/88

\section{DISCUSSION}

Portal hypertension is defined as an increase in the intravascular pressure within the portal vein of over $11 \mathrm{~mm}$ of mercury as measured directly or a splenic pulp pressure of over $16 \mathrm{~mm}$ of mercury. Pathophysiology of portal hypertension is due to rise in the portal pressure leads to splenomegaly and the development of natural portosystemic shunts at the following sites:

Lower end of the oesophagus and cardia through the gastro-oesophageal veins, the anal canal via the hemorrhoidal veins, in the falciform ligament via the umbilical veins, in the abdominal wall and retroperitoneum.(1)

We further classify based on the etiology as follows:

- $\quad$ Cirrhotic- e.g. biliary atresia, cystic fibrosis

- Non-cirrhotic

- Prehepatic- e.g. portal vein thrombosis, splenic vein thrombosis

- Intrahepatic

- Presinusoidal- e.g. noncirrhotic portal fibrosis, congenital hepatic fibrosis, sclerosing cholangitis, schistosomiasis

- Parasinusoidal- e.g. fatty liver, nodular hyperplasia, alcoholic hepatitis

- Postsinusoidal- e.g. veno-occlusive disease of liver, hepatic vein thrombosis

- Supra-hepatic-IVC web, (Budd-Chiari syndrome), constrictive pericarditis

EHPVO is the commonest cause of portal hyperintensity in children in India (80\%-90\% of cases of portal hypertension) and is defined as obstruction of the extrahepatic portal vein with or without involvement of the intrahepatic portal veins or splenic or superior mesenteric veins, isolated occlusion of splenic vein or superior mesenteric vein does not constitute EHPVO. The liver function tests in such patients are essentially normal. A confirmation of portal vein occlusion may be obtained by ultrasound demonstration of collateral venous channels at the porta hepatis replacing the portal vein. Approximately $40 \%-50 \%$ of these patients have a history of umbilical vein catheterization or abdominal sepsis in the neonatal period, but the venous occlusion in the majority appears to be congenital in origin. 
Coming to the hepatic causes most frequent cause of cirrhosis in childhood is biliary atresia. Besides this metabolic liver diseases, congenital hepatic fibrosis are other common medical conditions leading to cirrhosis of the liver. A liver biopsy shows bands of fibrous tissue joining the portal tracts and this condition may be associated with solid organ polycystic diseases.

Management of Portal Hypertension includes management of variceal hemorrhage, hypersplenism and other complications such as ascites, malabsorption, portal biliaemia.(2)

\section{A) Management of variceal bleed}

Medical measures include

- Airway stabilization.

- Hemodynamic resuscitation

- Pharmacologic therapy

- Vasopressin- the IV infusion of (0.2-0.4 units $/ 1.73 \mathrm{~m} / \mathrm{min}$ ), which may arrest the bleeding Vasopressin may be used alone or in combination with nitrates to reduce the portal venous pressure. Sideeffects related to systemic vaso-constriction like headache, nausea and abdominal cramps.

- Somatostatin-Octreotide, a long-acting analog of somatostatin has a plasma half-life of more than 1 hour. It reduces splanchnic blood flow and portal pressure with minimal side-effects, although the effectiveness of octreotide has been studied in a small number of children, its safety and side-effect profile have encouraged its use in cases of acute variceal bleeding.

- Endotherapy

a. Injection sclerotherapy

b. Variceal Banding

The strangulated varix, subsequently thromboses and sloughs. Usually up to three bands are applied at each session. Multi-band devices allow the application of several bands without the need for reloading. Treatment is performed initially at 1 to 2 weekly intervals, extending to monthly intervals once the larger varices are treated. The incidence of oesophageal stricture and systemic side-effects is lower with this treatment modality.

c. Glue Injection

Endoscopic variceal occlusion with tissue adhesives, such an n-butyl-cyanoacrylate or thrombin is more effective for acute fundal gastric varices in adults.

- Sengstaken-Blakemore (S-B tube) compression balloon- It may be life-saving when there is a failure of visualization of the varices due to overwhelming haemorrhage. Correct placement of the gastric balloon must be checked with X-ray control in order to avoid the inflation within the lumen of the oesophagus. Inflation of the gastric balloon and moderate prolonged traction achieved by securing the S-B tube to the side of the face with an adhesive tape is usually sufficient to stop the bleeding. It is rarely necessary to inflate the oesophageal balloon present on the standard instrument. Balloon deflation is performed 18 to 24 hours later and this is followed immediately with endoscopic variceal injection. (3)
Long term management of esophageal varices. ${ }^{(4)}$

- Endotherapy-Injection sclerotherapy, Transjugular Intrahepatic Porto-Systemic Shunt (TIPS)

- Surgery-Portosystemic shunts, liver transplantation.(5)

\section{MANAGEMENT OF COMPLICATIONS}

\section{B) Management of hypersplenism}

Symptomatic hypersplenism is found in less than $5 \%$ of the patients. Splenectomy along with shunt surgery DSRS may be indicated.

\section{C) Management of portal hypertensive gastropathy}

Propranolol had been studied in adults. Endoscopic thermal coagulation is not effective for diffuse bleeding. Liver transplantation is indicated for decompensated liver disease.

\section{D) Management of portal biliopathy}

Symptomatic portal biliopathy could be managed by endotherapy (Stone extraction, stricture dilatation, stenting) or surgery (Shunt surgery) portal biliopathy refers to abnormalities of the extrahepatic and intrahepatic bile ducts with or without anomalies of gallbladder wall in patients who have portal hypertension. The changes include indentations of paracholedochal collaterals on the bile duct, localized strictures, angulation of ducts, displacement of ducts and stones in the common bile duct and focal narrowing dilatations, irregular walls and clustering of intrahepatic branches in the hepatic ducts.

The approach to acute variceal hemorrhage in children is a stepwise progression from least invasive to most invasive. Surveillance endoscopy is predicated on the availability of an efficacious primary prophylactic therapy. Therefore, surveillance endoscopy and primary prophylaxis are not generally be indicated in children with portal hypertension who have not had a variceal bleed. Special medical and/or social circumstances in which an initial bleeding episode may be particularly dangerous could justify this approach.(6)

As previously stated, the main aim of therapy is to promptly treat the serious complication of hematemesis and massive blood loss. Recent reports indicate that esophageal varices in childhood are well controlled with either injection sclerotherapy or banding and if this fails portosystemic shunting would be the answer. Patients with portal vein obstruction and normal liver histology can be expected to live normal lives providing the esophageal varices are under control.(7)

\section{CONCLUSION}

The survival of the children with portal hypertension depends almost entirely on the etiology. Recent reports show that oesophageal varices in childhood are well controlled with either injection sclerotherapy or porto-systemic shunting and both methods have their advocates. Patients with portal vein obstruction and normal liver histology can be expected to live normal lives providing the oesophageal varices are under control.

\section{REFERENCES}

1. Bosch J.1 , Pizcueta P, Feu F, et al. Pathophysiology of portal hypertension. Gastroenterol Clin North Am. 1992 Mar;21(1):1-14. 
2. Samonakis DN, et al. Management of portal hypertension. Postgrad Med J 2004;80:634641 doi:10.1136/pgmj.2004.

3. Khurram Bari and Guadalupe Garcia-Tsao. Treatment of portal hypertension. Published online 2012 Mar 21;18(11):1166-1175.

4. Nina Dib, Frédéric Oberti, Paul Calès. Current management of the complications of portal hypertension: variceal bleeding and ascites. CMAJ May 9 , 2006;174(10):1433.

5. Yoshida H. ${ }^{1}$, Mamada Y, Taniai N, et al. New trends in surgical treatment for portal hypertension. Hepatol Res. 2009 Oct;39(10):1044-51.
6. Roberto Gugig and Philip Rosenthal. Management of portal hypertension in children. World J Gastroenterol. 2012 Mar 21;18(11):1176-1184. Published online 2012 Mar 21. doi: 10.3748/wjg.v18.i11.1176 pmcid: pmc 3309906.

7. Mohamed A El Guindi, Hisham M Nazer. Portal hypertension and esophageal varices. Abdelaziz Y Elzouki (ed). Textbook of clinical pediatrics. Doi 10.1007/978-3-642-02202-9_214, \# Springer-Verlag Berlin Heidelberg 2012. 